\title{
ON THE WEAK LIMIT OF RAPIDLY OSCILLATING WAVES
}

\section{CHIERCHIA, N. ERCOLANI, AND D. MCLAUGHLIN}

I. Introduction. Conservative, dispersive waves form, and propagate, as packets of rapidly oscillating wavetrains. The mathematical description of this physical process is rather complete for linear waves, but is still in its infancy for nonlinear waves. One mathematical formulation of this problem [1] is as follows: Given a rapidly oscillating nonlinear wave $U(x, t)$, (i) characterize and (ii) derive evolution equations for the weak limit of $U$ (as the wavelengths of the oscillations vanish).

Near-integrable waves [2-6] provide interesting and rich examples for the study of the propagation of rapid, nonlinear waves. One formal approach [7-9] is to construct an asymptotic representation of the wave $U(x, t)$, from which one explicitly calculates both the weak limit and the evolution equations that it satisfies. In the near-integrable framework [8], this approach yields a local (in space $x$ and time $t$ ) representation of the wave in the form

$$
U^{e}(x, t)=W_{N}\left(\frac{\theta(x, t)}{\varepsilon} ; \kappa(x, t), \omega(x, t)\right)+O(\varepsilon),
$$

where for each $\kappa, \omega$ the function $W_{N}(\theta)$ is a real-valued function on the $N$-torus $\mathrm{T}^{N} \equiv \mathrm{R}^{N} / 2 \pi \mathrm{Z}^{N}$,

$$
W_{N}(\cdot, \kappa, \omega): \mathrm{T}^{N} \rightarrow \mathrm{R} .
$$

The $N$-vectors $\theta, \kappa, \omega$ are real-valued functions of $x$ and $t$ related by

$$
\begin{aligned}
\frac{\partial}{\partial x} \theta_{i} & =\kappa_{i}, \\
\frac{\partial}{\partial t} \theta_{i} & =\omega_{i}, \quad i=1,2, \ldots, N .
\end{aligned}
$$

Equations (I.2) imply that

$$
\frac{\partial}{\partial t} \kappa_{i}=\frac{\partial}{\partial x} \omega_{i}, \quad i=1,2, \ldots, N .
$$

Received February 25, 1987. 
One must then derive [8] $N$ additional equations of the form

$$
\frac{\partial}{\partial t} \omega_{i}=\frac{\partial}{\partial x} F_{i}(\kappa, \omega), \quad i=1,2, \ldots, N
$$

Equations (I.3a, b) are the desired evolution equations.

A first step toward converting this formal construction into a rigorous mathematical derivation is to assume that $U^{\varepsilon}$ is of form (I.1), and then to establish that

$$
\begin{gathered}
\underset{\substack{\text { weak-lim } \\
\varepsilon \downarrow 0}}{\varepsilon}(x, t)=\bar{U}(x, t), \\
\bar{U}(x, t) \equiv \frac{1}{(2 \pi)^{N}} \int_{T^{N}} U^{\varepsilon}(\theta ; \kappa(x, t), \omega(x, t)) d \theta .
\end{gathered}
$$

In section II of this paper we prove a version of (I.4) under suitable assumptions.

We remark that in the simple phase case $(N=1)$, this result is classical; however, for the general case $(N>1)$, a "nonresonance condition" about the $\kappa$ 's is required. In our principal applications (see [9], section IV and appendix A) we have viewed modulating wavetrains as evolving in $t$ on a (formal) space of functions of $x$. Therefore the theorem we formulate below is independent of $t$ except insofar as the local nonresonance condition is or is not satisfied at a given $t$. As we explain in section III, we believe that the nonresonance condition formulated in this paper is basically optimal.

One fascinating feature in the propagation of rapidly oscillating, integrable, nonlinear waves is that the number of phases $N$ can change in space and time $[3,8,2]$. In section III of this paper we discuss connections between the breakdown of the nonresonance condition and curves in the $x$ - $t$ plane across which the number $N$ of phases changes.

\section{An averaging theorem.}

Definition. A curve $\kappa: x \in \mathrm{R} \rightarrow \kappa(x) \in \mathrm{R}^{n}$ is said to be locally nonresonant if any finite interval of $R$ can be decomposed in a countable union of disjoint intervals $I_{n}$ so that $\kappa$ is $C^{1}$ on the interior of $I_{n}$ and for any $\nu \in Z^{N} \backslash\{0\}$ the function

$$
x \in I_{n} \rightarrow \kappa(x) \cdot \nu \equiv \sum_{i=1}^{N} \kappa_{i}(x) \nu_{i}
$$

has at most a finite number of zeros.

Now, let

$$
(\theta, x) \in \mathrm{T}^{N} \times \mathrm{R} \rightarrow W(\theta, x)
$$


be a $C^{1}$ function such that for any finite interval $I$

$$
\sum_{\nu \in Z^{N}} \int_{I}\left|\hat{W}_{\nu}(x)\right| d x<\infty
$$

where the circumflex denotes Fourier coefficients:

$$
\hat{W}_{\nu}(x) \equiv \frac{1}{(2 \pi)^{N}} \int_{T^{N}} W(\theta, x) e^{-i \nu \cdot \theta} d \theta
$$

ThEOREM. If $\theta(x)$ is a curve in $\mathrm{T}^{N}$ with $\theta^{\prime}(x) \equiv(d / d x) \theta \equiv \kappa(x)$ locally nonresonant, then

$$
\underset{\varepsilon \downarrow 0}{\text { weak-lim }} W\left(\frac{\theta(x)}{\varepsilon}, x\right)=\frac{1}{(2 \pi)^{N}} \int_{T^{N}} W(\theta, x) d \theta \equiv \hat{W}_{0}(x) .
$$

That is, for any smooth function $\phi$ with compact support,

$$
\lim _{\varepsilon \downarrow 0} \int W\left(\frac{\theta(x)}{\varepsilon}, x\right) \phi(x) d x=\int \hat{W}_{0}(x) \phi(x) d x .
$$

Proof. We divide the proof into four steps.

Step 1. Proving (II.2) is clearly equivalent to showing that

$$
\lim _{\varepsilon \downarrow 0} \int_{I} W\left(\frac{\theta(x)}{\varepsilon}, x\right) d x=\int_{I} \hat{W}_{0}(x) d x
$$

for any finite (closed) interval $I$. Furthermore, because of the nonresonancy assumption, we can assume that on $I, \kappa \cdot \nu$ has at most a finite number of zeros for $\nu \in Z^{N} \backslash\{0\}$.

Step 2. Now we expand in Fourier series. Because of (II.1), for any $\eta>0$ there exists a positive integer $M$ such that (denoting $\left.|\nu| \equiv \sum_{i=1}^{N}\left|\nu_{i}\right|\right)$

$$
\sum_{|\nu|>M} \int_{I}\left|\hat{W}_{\nu}(x)\right| d x<\eta
$$

Therefore, because of the arbitrariness of $\eta$, the theorem will follow from

$$
\lim _{\varepsilon \downarrow 0} \int_{I} \hat{W}_{\nu}(x) e^{i(\nu \cdot \theta(x) / \varepsilon)} d x=0
$$

for any $\nu$ with $0<|\nu| \leq M$. 
Step 3. Let $\phi(x)$ be any $C^{1}$ function on a closed interval $I$ with $\phi^{\prime}(x) \neq 0$, and let $f$ be continuous on $I$. Then

$$
\lim _{\varepsilon \downarrow 0} \int_{I} e^{i(\phi(x) / \varepsilon)} f(x) d x=0 .
$$

This follows immediately from the Riemann-Lebesgue lemma if one notices that the integral above can be written as

$$
\int_{\phi(I)} e^{i(\phi / \mathrm{e})} f(x(\phi)) \frac{d x}{d \phi} d \phi,
$$

where $x(\phi)$ denotes the inverse function of $\phi(x)$.

Step 4. Let $\nu$ be fixed as above, i.e., $0<|\nu| \leq M$. Then by step 1 there is at most a finite number of zeros of $x \in I \rightarrow \kappa(x) \cdot \nu$. Denote them $\hat{x}_{1}<\hat{x}_{2}<\cdots$ $<\hat{x}_{m}$. Because of (II.1), for any $\sigma>0$ there exists a $\rho>0$ such that (denoting $I_{\rho}$ the set $\left.I \cap \bigcup_{j=1}^{m}\left(\hat{x}_{j}-\rho, \hat{x}_{j}+\rho\right)\right)$ one has

$$
\int_{I_{\rho}}\left|\hat{W}_{\nu}(x)\right| d x<\sigma .
$$

So to prove (3), it is enough to show that

$$
\lim _{\varepsilon \downarrow 0} \int_{I \backslash I_{\rho}} \hat{W}_{\nu}(x) e^{i((\nu \cdot \theta(x)) / \varepsilon)}=0 .
$$

But this is immediate from step 3, since $I \backslash I_{\rho}$ is a finite union of intervals over which $\theta^{\prime} \cdot \nu$ is bounded away from 0 .

III. Comments on the nonresonance condition. An obvious example of locally nonresonant curves $\kappa$ is given by certain piecewise constant functions

$$
\kappa(x)=\sum_{n} \kappa_{n} \chi_{I_{n}}(x)
$$

where $\left(I_{n}\right)_{n \in \mathrm{N}}$ is a partition of $\mathrm{R}$ in finite intervals, $\chi$ denotes characteristic functions, and $\kappa_{n}$ are rationally independent vectors in $R^{N}$. Another elementary class of nonresonant curves can be built up by patching together real analytic curves $\kappa^{(n)}$ having functionally independent components. In this case $x \rightarrow$ $\kappa^{(n)}(x) \cdot \nu$ will locally have a finite number of zeros for any real vector $\nu$. More abstractly, it would not be difficult to show that our condition is, in a suitable sense, generic.

It is also easy to understand what happens in case of resonance. Two types of phenomena can occur: the weak limit may not exist at all (this is trivially seen to 
be the case for constant $\theta(x))$, or the limit may differ from the simple average. For example, let $\theta(x)$ be the canonical projection (from $R^{N}$ to $\mathrm{T}^{N}$ ) of a straight line $x \rightarrow \nu_{0} x$, with $\nu_{0} \in Z^{N} \backslash\{0\}$. Then, from the preceding section, one concludes that

$$
\underset{\varepsilon \downarrow 0}{\operatorname{weak}-\lim } W\left(\frac{\theta(x)}{\varepsilon}, x\right)=\sum_{\nu \in \nu_{0}^{\perp} \cap Z^{N}} \hat{W}_{\nu}(x),
$$

where $\nu_{0}^{\perp} \equiv\left\{\mu \in Z^{N}: \mu \cdot \nu_{0}=0\right\}$.

The zero dispersion limit for solutions of the $\mathrm{KdV}$ equation was shown to exist as a weak limit in [3]. This work indicates that the weak limit may be characterized in terms of a hierarchy of equations indexed by $N$. When $N=1$, these are Whitham's equations; for $N>1$ they are described in [5]. These equations are hyperbolic and can be written in Riemann invariant form

$$
\lambda_{i t}(x, t)-S_{i}\left(\lambda_{i}, \vec{\lambda}\right) \lambda_{i x}(x, t)=0, \quad i=1, \ldots, 2 N+1
$$

It is natural to attempt to represent the weak limit as the limit of $N$-phase waves $[1,8]$, as indicated in (I.4). There will be curves in the $x$ - $t$ plane across which the number of phases, $N$, changes. If one solves for $t=t(x)$ implicitly from $\lambda_{i}(x, t)=$ constant, for some $i$, then inserting $t(x)$ into (I.4) reduces the equation to the form (II.2). Along curves of discontinuity for $N(x, t)$, the nonresonance condition will be violated. However, it seems reasonable that for nice initial data this condition is violated only along curves where $N(x, t)$ changes. If such is the case, then the Riemann invariant trajectories are locally nonresonant curves. This would at least make the problem of characterizing the weak limit in terms of $N$-phase limits well posed, i.e., (I.4) would be uniformly valid in the $x$ - $t$ plane.

\section{REFERENCES}

1. R. Diperna, "Measure valued solutions to conservation laws," to appear, Proc. of Conf. on Rapid Oscillation Theory, Minnesota, ed. D. Kenderlehrer and M. Slemrod (1985).

2. N. Ercolani, M. G. Forest, and D. W. McLaughlin, "Oscillations and instabilities in near integrable PDE's," Proc. of AMS-SIAM Conference on Nonlinear Evolution Equations, Santa $\mathrm{Fe}$, ed. D. Holm, J. M. Hyman, and B. Nicolaenko (1985).

3. P. D. LAX AND C. D. LeVermore, The small dispersion limit for the KdV equation, I, Comm. Pure Appl. Math. 36 (1983), 253-290; II. Comm. Pure Appl. Math. 36 (1983), 571-594; III, Comm. Pure Appl. Math. 36 (1983), 809-829.

4. S. Venakides, "The zero dispersion limit of the $\mathrm{KdV}$ equation with periodic initial data," to appear, Proc. of Conf. on Rapid Oscillation Theory, Minnesota, ed. D. Kenderlehrer and M. Slemrod (1985).

5. H. FlaschKa, M. G. Forest, and D. W. McLaughlin, Multiphase averaging and the inverse spectral solution of the Korteweg-de Vries equation, Comm. Pure Appl. Math. 33 (1980), 739-784. 
6. M. G. FOREST AND D. W. MCLAUGHLIN, Modulations and sinh-Gordon and sine-Gordon wavetrains, Stud. Appl. Math. 68 (1983), 11-59.

7. D. W. Mclaughlin, G. Papanicolaou, and L. Tartar, "Weak limits of semilinear conservation laws with oscillating data," to appear, Proc. of Con. on Rapid Oscillation Theory, Minnesota, ed. D. Kenderlehrer and M. Slemrod (1985).

8. D. W. MCLAUGHLin, "On the construction of a modulating, multiphase wavetrain for a perturbed KdV equation," to appear, Proc. of Conf. on Rapid Oscillation Theory, Minnesota, ed. D. Kenderlehrer and M. Slemrod (1985).

9. N. Ercolani, M. G. Forest, D. W. Mclaughlin, and R. Montgomery, "Hamiltonian structure for the modulation equations of sine-Gordon wavetrain," Univ. of Arizona preprint (1986).

Chierchia: Dipartimento di Matematica, IIA Universitá di Roma, 00173 Rome, Italy

Ercolani, Mclaughlin: Department of Mathematics, University of Arizona, Tucson, ARIZONA 85721 